\title{
Formulation, Development and Validation of a Wound Healing Herbal Ointment from Extracts of Bidens pilosa and Aloe barbadensis
}

\author{
Sandra Namunana ${ }^{1}$, Stephen Lutoti ${ }^{2,3 *}$,Grace Nyamaizi ${ }^{1}$, Gerald Agaba ${ }^{1}$, Irene Apun ${ }^{1}$, \\ Charles Ssebunnya ${ }^{1}$, Gladys Mercy Tenywa ${ }^{2}$,Raphael Wangalwa ${ }^{2}$, Bruhan Kaggwa ${ }^{1,2}$, \\ Pakoyo Fadhiru Kamba ${ }^{1}$, David Musoke-Muweke ${ }^{3}$, Patrick Ogwang Engeu² \\ ${ }^{1}$ Makerere University, Department of Pharmacy, School of Health Sciences, Kampala, Uganda \\ ${ }^{2}$ Pharm-Biotechnology and Traditional Medicine Center of Excellence for Eastern and Southern Africa Mbarara \\ University of Science and Technology, Department of Pharmacy, Faculty of Medicine, Mbarara, Uganda \\ ${ }^{3}$ Gulu University, Department of Pharmacology and Therapeutics, Gulu, Uganda
}

*Corresponding Author: Stephen Lutoti, Pharm-Biotechnology and Traditional Medicine Center of Excellence for Eastern and Southern Africa Mbarara University of Science and Technology, Department of Pharmacy, Faculty of Medicine, P.O Box 7062, Mbarara, Uganda, Tel: +256-782764180; E-mail: lutoti@yahoo.com

Received: 07 April 2018; Accepted: 16 April 2018; Published: 18 April 2018

\begin{abstract}
Various studies have been done to assess the wound healing potential of Aloe barbadensis and Bidens pilosa. Plant materials/ extracts that have been used in most of those studies have shown superior/ comparable efficacy with respect to positive controls used. However, there remains a big gap concerning standardisation of doses to use since there is no information on how the doses used in these experiments are determined. This study aimed at formulating and preparing a herbal ointment (BILOE-8) from Aloe barbadensis and Bidens pilosa; establishing the quality, wound healing efficacy and toxicity profile of the prepared herbal ointment. Ethanolic extracts of the leaves of the study plants were prepared and screened for presence of alkaloids, flavonoids (B. pilosa), and anthraquinones (A. barbadensis). Formulation of BILOE-8 was by fusion. Standard tests for ointment quality and microbiological stability were done. An experimental controlled study was carried out to determine the efficacy of BILOE-8 which appears black with a smooth surface, is consistent, spreads easily on dry skin, washable but leaves a green stain that disappears with time. No observable physical, chemical, and microbiological changes were observed. BILOE-8 had the fastest rate of wound reduction, and the shortest epithelialization and healing times compared to the other treatments. No cutaneous changes were observed within 24 hours. A herbal ointment formulated from Aloe
\end{abstract}


barbadensis and Bidens pilosa is therefore an efficacious wound healing remedy that should be considered as an alternative medicine for wound management.

Keywords: Wound healing; Herbal medicine; Formulation; Aloe barbadensis; Bidens pilosa

\section{Introduction}

Wounds and burns can be major causes of physical disability and may lead to loss of many productive hours. Essentially, wounds are the disruption of functional continuity and anatomical structure of cells and tissues at the sites of injury. They can be caused by insults to the tissue by physical, chemical, microbiological or immunological processes [1]. It has been estimated that 14 million people suffer from wounds and burns annually with over 80 percent of these living in low and middle income countries [2]. However this may be under reported since wounds are a daily occurrence that can happen to anyone at any time and people do not always seek medical attention for simple wounds and burns.

Man has in situ capabilities of healing wounds through continuous tissue repair and tissue regeneration [3]. The curing of acute and chronic wounds and wounds from burns proceeds through the common basic phases of hemoptysis, inflammation, proliferation, fibriplasia, collagen deposition, epithelialization, contraction, remodeling and maturation [4].

The capabilities mentioned above can be impaired or slowed down by factors such as age, dessication, necrosis, pressure at wound site, body type (e.g. obesity), chronic diseases (e.g. cancer, peripheral vascular disease, diabetes mellitus, etc.), immunosuppression, nutritional status, vascular insufficiency, and infection with microbes such as bacteria [5]. There is, therefore, a need for intervention in form of treatment.

In Uganda, wounds and burns are mainly managed by antimicrobial agents commonly Neomycin Sulphate (NS) cream and Silver Sulfadiazine (SSD) cream [6] respectively. Based on our clinical practice observations, it is common practice to manage wounds and burns using metronidazole powder or intravenous solutions in Mulago hospital, even though this has not been documented for routine practice. This 'overt' use of conventional medicine has some shortfalls: hypersensitivity to some of the components in these drugs, expense, development of resistance, and serious adverse effects that may occur when absorbed systemically, which is almost unavoidable when applied on open wounds.

Traditional medicine is an integral part of health care. One study quotes that according to the WHO Traditional Medicine Strategy 2002-2005,

"High per capita distribution of TCAM practitioners in developing countries is an important reason for the widespread use of TCAM. WHO report cites example of Uganda, Tanzania and Zambia where the ratio between populations to healer is 1:200 to 1:400 while the ratio of allopathic practitioners to population is 1:20,000.” [7]. 
According to a recent source, these ratios have not changed [8]. In addition, it is estimated that usage of traditional medicine in the health care system in Uganda is $60 \%$ [8]. Traditional medicine is used globally and is of rapid growing economic importance [9]. In developing countries, the use of herbs is not only an alternative to the mainstay, which is western medicine, but also the most easily accessible and affordable treatment [10].

Herbal treatment for wounds provides fibro-genetic and concentration of collagen resulting in faster wound healing [4]. The growing popularity of natural and herbal medication, easy availability of raw materials, cost effectiveness and paucity of reported adverse reactions prompted us to formulate a polyherbal topical preparation containing extracts from Aloe barbadensis and Bidens pilosa and assess its wound healing ability on experimentally induced cutaneous wounds in rat models.

Aloe barbadensis (Liliaceae), popularly known as Aloe vera has antibacterial, anti-inflammatory and analgesic properties very useful in wound management. It is native to North Africa, the Mediterranean region of southern Europe, and the Canary Islands [11]. It's a hardy perennial semi tropical plant which can also thrive in drought prone areas [12].

Bidens pilosa (Asteraceae) is native to South America but is now distributed all over the world, especially in tropical and subtropical regions. Its wound healing activity has been established [13]. In Uganda, there is evidence that these two herbs (Aloe barbadensis and Bidens pilosa) have traditionally been used in wound healing. Research has also proven their efficacy in this regard, and this justifies the formulation of this product (BILOE-8).

\section{Materials and Methods}

\subsection{Study design}

This was a controlled experimental study.

\subsection{Materials used in the study}

Leaves of Bidens pilosa ,the leaves of Aloe barbadensis; Fifteen Albino rats (obtained from College of Veterinary medicine, animal resources and biosciences in Makerere University); Ethanol (90\%), Sulphuric acid, ammonia, chloroform, Mayer's reagent, sodium hydroxide, soyabean Casein Digest Agar, distilled water; Wool alcohol, Hard paraffin, Cetostearyl alcohol, White Soft Paraffin, Vitamin E; Lidocaine 2\% for injection, Diclofenac (75 mg/3 mL) for injection, Neomycin Sulphate 1\% cream ; Cotton wool, control microorganisms - Staphylococcus aureus, rodent pellets (food); Knife, $2 \mathrm{~mL}$ syringes and needles, surgical blades, pair of forceps, pair of scissors, disposable gloves, conical flasks, mortar and pestle, Petri dishes, Rotary Evaporator, incubator, thermometer, tray, refrigerator, autoclave, spatulas, inoculating wire loop, filter paper, filter funnels, measuring cylinders, electronic weighing balance and oven. 
2.2.1 Plant collection: Fresh leaves of Bidens pilosa was collected from convenient bushes around Makerere University. Fresh leaves of Aloe barbadensis were collected from a flower garden in Kyebando, about 4.3 miles from the centre of Kampala, the capital of Uganda. Photographs of plants in their natural habitat were taken. The plant materials were separately placed in clean polythene bags, sealed and given an identification numbers. The collected plant materials were labelled with the following details: botanical name of plant, location of collection, part that has been collected, date, time of collection and identification number and Herbarium specimen deposited at the Department of Pharmacy, Makerere University.

2.2.2 Plant extraction site: The extraction of phytochemicals with activity from parts of the plants was carried out in the Pharmacognosy laboratory at the Pharmacy Department, College of health sciences, Makerere University.

Preparation of the ethanolic extraction of Aloe barbadensis: The leaves were shed-dried for a week. The large leaves were cut into smaller potions that could easily be pounded. The dry leaves were pulverised using a blender. Carefully, $300 \mathrm{~g}$ of the powder was weighed and extraction was done by cold maceration in $750 \mathrm{~mL}$ of $90 \%$ ethanol for 72 hours. Thereafter, the macerate was filtered using filter paper and the extract was concentrated at $85^{\circ} \mathrm{C}$ using a rotary evaporator to obtain a viscous liquid. Excess water was evaporated using a hot air oven to obtain a powder.

Preparation of the Ethanolic Extract of Bidens pilosa: The leaves were shed-dried for a week. The dry leaves were pulverised mechanically using a mortar and pestle. Carefully, $750 \mathrm{~g}$ of the powder was weighed and extraction was done by cold maceration in $1500 \mathrm{~mL}$ of $90 \%$ ethanol for 72 hours. The macerate was filtered using filter paper and the extract was concentrated at $85^{\circ} \mathrm{C}$ using a rotary evaporator to obtain a viscous solid. This was then left in a dark place to evaporate the remaining ethanol [13].

2.2.3 Phytochemical screening of the extracts: The plant extract of Bidens pilosa was screened for presence of compounds that are essential in wound healing (alkaloids and flavonoids) following methods described by Hossain et al. [14]. For testing presence of alkaloids, Ammonia solution $(3 \mathrm{~mL})$ was added to Bidens pilosa powder $(1 \mathrm{~g})$ and left to stand for a few minutes. Afterwards, chloroform $(10 \mathrm{~mL})$ was added, and the mixture was shaken and filtered. The chloroform was evaporated by water bath. $3 \mathrm{~mL}$ of Mayer's reagent were then added [14].

Aloe barbadensis extract was screened for presence of anthraquinones by method described by Evans. Briefly, the test material was boiled with $1 \mathrm{ml}$ of Sulphuric acid in a test tube for five minutes, after which it was filtered (while hot). The filtrate was cooled and shaken with an equal volume of chloroform. The lower layer of chloroform was then separated and shaken with half of its volume of dilute ammonia.

2.2.4 Positive Control Drugs: The positive control drugs used in this experiment were obtained from Friecca Pharmacy, a private community pharmacy that is registered by the National Drug Authority (NDA) in Uganda. Verification was done concerning registration of the brand of drug to be used, physical examination to be done and 
confirmation with NDA Drug control laboratory on whether the batch purchased in the pharmacy was analysed by NDA.

2.3 Formulation and preparation of the ointment

The following formulae were used (Table 1).

\begin{tabular}{|l|l|l|l|}
\hline Ingredients & $\begin{array}{l}20 \text { g 5\% Aloe } \\
\text { barbadensis ointment }\end{array}$ & $\begin{array}{l}\text { 20 g 15\% Bidens } \\
\text { pilosa ointment }\end{array}$ & $\begin{array}{l}\text { g0 combination } \\
\text { ointment (BILOE-8) }\end{array}$ \\
\hline Active Ingredients & - & $1 \mathrm{~g}$ \\
\hline Ethanolic extract of Aloe barbadensis & $1 \mathrm{~g}$ & $3 \mathrm{~g}$ & $3 \mathrm{~g}$ \\
\hline Ethanolic extract of Bidens pilosa & - & $1 \mathrm{~g}$ & $1 \mathrm{~g}$ \\
\hline Excipients & $1 \mathrm{~g}$ & Qs & Qs \\
\hline Vitamin E & Qs &
\end{tabular}

Table 1: Formulation and preparation of the ointment.

All ingredients were weighed using an electronic weighing balance. Simple ointment was first prepared. Hard Paraffin was placed into an evaporating dish and melted over a water bath. The dish was removed from heat and the other ingredients were added in descending order of melting point until all were melted in (the order being Cetostearyl Alcohol next, then Wool Fat, and lastly White Soft Paraffin). The mixture was continuously stirred to ensure homogeneity, but at the same time gently to avoid incorporation of excess air.

Vitamin E and plant substances (ethanolic extract of Bidens pilosa and/or Aloe barbadensis gel) were weighed and mixed together before a sufficient amount of Simple Ointment BP was added to make up to the required quantity while stirring $[15,16]$.

2.3.1 Packaging: The ointments were packaged in small clear plastic containers with contents of $20 \mathrm{~g}$ net weight.

2.3.2 Weight variation: The filled containers were weighed and the average weight was calculated. This was compared with the expected average weight from theoretical calculation based on knowledge of weight of empty containers.

2.3.3 Labeling: The labels that were placed on the container of the ointment contain the following information: Name of the product, net Weight, ingredients- medicinal and non-medicinal, indication, directions for use, caution, date of manufacture, expiry date, batch number, manufacturer and their address. 


\subsection{Quality control testing of the study ointment}

The following qualitative tests were carried out on the prepared ointment as described by Aulton [17] and (UNC Eshelman School of Pharmacy) to depict of quality of prepared ointments.

2.4.1 Appearance: The general appearance of the formulated ointment was observed and recorded. Qualities included colour, granular or lumpy surface

2.4.2 Consistency: A small amount of ointment was slowly rubbed between the thumb and fore finger to gauge consistency of the ointment.

2.4.3 Spreadability: A small amount of ointment was rubbed on the back of the hand and the ease with which it is spread over the skin was noted.

2.4.4 Washability: A small amount of ointment was rubbed on the back of the hand, after which it was washed off with warm water.

\subsection{Stability of study ointments}

2.5.1 Physical and chemical stability: BILOE-8 in its packaging was observed every three (3) days for physical changes that were visible to the naked eye. The ointments were subjected to the following conditions: open and exposed to air, and closed at room temperature in both light and darkness.

2.5.2 Microbiological stability: Dextrose Agar was used in the determination of microbiological stability of the formulated product. The test explained below was done in triplicate, both for the positive control and for the combination product. For preparation of the agar, manufacturer's instructions available on the label were followed. For testing, Staphylococcus aureus was cultured for 48 hours as a positive control (agar growth promotion test). The study ointment (BILOE-8) was applied to culture media and inspected for growth after 24- 48 hours.

\subsection{Test for efficacy}

Fifteen (15) albino rats of more than 8 weeks old, and weight range 123-213 g (average $165.225 \mathrm{~g}$ ) were used. Prior to starting the experiment, the rats were randomized into six sets of three rats each using Online Research Randomiser. After that, the rats were labeled in the order that they were picked and then placed into their assigned groups. The groups were as follows (Table 2): 


\begin{tabular}{|l|l|l|l|l|l|l|}
\hline Model & Test & No. & Positive Control & No. & Negative Control & No. \\
\hline \multirow{3}{*}{ Fresh Wound } & Bidens pilosa & 3 & NS Cream & 3 & Simple Ointment BP & 3 \\
\cline { 2 - 3 } & Aloe barbadensis & 3 & & & & \\
\cline { 2 - 4 } & BILOE-8 & 3 & & & & \\
\hline
\end{tabular}

Table 2: Randomized rats into six sets of three rats each using Online Research Randomiser.

The mid dorsal region of the study rats was shaved, and a circular area with a diameter of about $1 \mathrm{~cm}$ was mapped out. This area was disinfected with $70 \%$ ethanol. The forceps and pair of scissors that were used were disinfected prior to use to prevent wound infection. A full thickness wound was cut out along the margins of the mapped out area. Where bleeding occurred, it was arrested using cotton wool. The wounds were cleaned with distilled water before application of any ointment. Ointments were applied twice daily till complete healing.

For anaesthesia, the rats were anaesthetised using SC Lidocaine hydrochloride $2 \%$ dosed at $2.5 \mathrm{mg} / \mathrm{kg}$ [18]. For analgesia, the rats were injected with IM Diclofenac $100 \mathrm{mg} / \mathrm{kg}$ preoperatively and every 24 hours postoperatively for 3 consecutive days as earlier described by Hassan et al. [13].

Healing time was determined as the time taken for the wound to heal completely. Wound size $(a b)$ was measured using Vernier callipers and a $30 \mathrm{~cm}$ ruler for verification. Measurements were done every other day until complete healing. Area was then calculated as:

$$
\frac{a b \pi}{4}
$$

where $a$ and $b$ were horizontal and vertical diameters of the circle. The rate of wound contraction was measured as the percentage rate of wound closure with respect to three selected days. Time for Complete Epithelialisation was the time taken for a layer of epithelium to form over a denuded surface.

\subsection{Test for acute dermal toxicity}

Three rats were assigned to this group. The site of application (mid dorsal region) was shaved and cleaned with distilled water, after which a large quantity $(300 \mathrm{mg} / \mathrm{kg}$ ) of the combination product (BILOE-8) was applied. The area was inspected within a period of 24 hours for any cutaneous changes such as erythema, swelling, and development of vesicular eruptions.

2.7.1 Quality assurance: Good Laboratory Practice (GLP) was ensured at all stages of the study and this included sterilization of all instruments and glassware used for microbial analysis, All reagents and positive control drugs were sourced from certified suppliers, Proper labeling of all reagents, materials, media and chemicals was done, Calibrated weighing balances and instruments were used for measuring various reagents, chemicals, media and 
materials, Documented procedures that had previously been used and demonstrated accuracy and precision of results were used.

2.7.2 Data management: The data collected in the study was recorded in a logbook.

2.7.3 Statistical analysis: The data generated in the study was summarized into tables and graphs, after which it was analysed using ANOVA, means, standard deviations, 99.5\% confidence intervals, and $\mathrm{p}$ values.

2.7.4 Ethical considerations: Ethical clearance for this study was obtained from the Makerere University School of health sciences IRB housed at Makerere University Pharmacy Department. After the study, the animals were given back to Makerere UniversityCollege of Veterinary Medicine, Animal resources, and Biosecurity .

\section{Results and Discussion}

\subsection{Extraction, product formulation and preparation}

Herbal ointments from extracts for A. barbadensis and B. pilosa were successfully formulated, packaged in unit plastic containers of $20 \mathrm{~g}$ net weight, and labelled. The backbone of the formula used was Simple Ointment BP. This is an ointment preparation based on oleaginous bases into which the active ingredients were incorporated. We used the fusion method to prepare the ointment. This was to ensure even distribution of ingredients within the formulation. The melting points of the ingredients used to make the simple ointment base were taken into consideration since they are the basis by which these ingredients are added in a specific order. Cetostearyl Alcohol BP: 49-56 ${ }^{\circ} \mathrm{C}$ (BP 1988, p 111) [19], Hard Paraffin BP: 50-61 ${ }^{\circ} \mathrm{C}$ (BP 1988, p 415) [19], White/Yellow Soft Paraffin BP: $38-56^{\circ} \mathrm{C}$ (BP 1988, p 416) [19], Wool Fat BP: $38-44^{\circ} \mathrm{C}[15,16]$. From this knowledge, the addition of the ingredients was in the order of hard paraffin, cetostearyl alcohol, wool fat and lastly white soft paraffin. BILOE-8 exhibited the desired properties of an ointment namely consistency, spreadability, and physicochemical stability. However, its black appearance and staining properties may make it cosmetically unacceptable. Also, like other ointments, it cannot be applied onto a moist surface, for example an exuding wound.

Hydrocarbon bases were chosen for this formulation so as to come up with an oleaginous preparation with good occlusive power to prevent moisture loss from the skin and hence aid healing. This is the basis of their emollient action. The bases included Hard Paraffin, White Soft Paraffin, Cetostearyl alcohol, and Wool Fat. White Soft Paraffin is also used as the diluent in this formulation due to its little to no toxicity when used in large amounts for topical application. Vitamin $\mathrm{E}$ as a fat soluble antioxidant was employed in this formulation to prevent the oxidation of the fatty bases, hence preventing spoilage of the ointment.

In the packaging of an ointment, aluminium and tin tubes, as well as plastic containers may be used. However plastic is preferred because aluminium and tin may contaminate the product, and make it unsuitable for use. In fact, various pharmacopoeial tests limit the extent of such contamination [17]. In this study, the ointments were packaged in small clear plastic containers with contents of $20 \mathrm{~g}$ net weight. 
To ensure quality, the factors that are evaluated for a new ointment during development and storage include Stability of the active ingredients, stability of the adjuvants, rheological properties (consistency, viscoelasticity, extrudabilty), Loss of volatile components, including water, Phase changes- inhomogeneity, bleeding, cracking, Particle size distribution of dispersed phase, Apparent pH , Particulate contamination [17]. For this study, the quality tests that were carried out included appearance, consistency (uniformity and rheology), spreadability, and washability. Biloe-8 ointment appeared black with a smooth surface, was found to be consistent (had no clumps or particles), easy to spread (especially on dry skin) and left a green stain on washing, that disssapeared within a day.

In terms of Physical and chemical stability of Biloe- 8 ointment, there were no observable changes under all tested conditions for three months. Shelf life of the ointment can be determined by means of accelerated stability tests at elevated temperatures and the Arrhenius relationship. The ointment may bleed as its matrix contracts and squeezes out, and the high temperature can produce and accelerate such adjustments, and this would have helped to estimate the shelf life. This was, however, not possible due to limitations of time and accessibility of stability chambers. According to NDA Guidelines for regulation of traditional medicines in Uganda, all local products shall have a shelf life of not more than one year. As for the Microbiological stability, BILOE-8 does not support growth of Staphylococcus aureus.

Owing to the low yields obtained during the extraction process (Yield of extract for A. barbadensis was 3.55\% while that for B. pilosa was $7.76 \%$ ), there is requirement for large amounts of raw materials. Anthraquinones are substances known to cause irritation and their amounts need to be controlled. The Weight variation between theoretical and actual calculation of the weight of the filled containers was $1.04 \%$.

\subsection{Relative comparison of changes in wound area for the different ointment treatments}

Within the first three days of treatment, all slopes on the graph appear to have a similar gradient. This could be due to natural processes taking charge, in addition to the inherent wound healing effects of the treatments. The observed activities are due to presence of Alkaloids and flavonoids (detected in the Bidens pilosa extract) and Anthraquinones (detected in small quantities in the Aloe barbadensis extract) which were the active ingredients in the formulation of BILOE-8 ointment.

Based on Figure 1, BILOE-8 ointment seems to quicken the wound reduction process relative to the others as evidenced by the deeper dip of its slope within this time period. On the other hand, ABO seems to slow the rate in the beginning, judging from the difference in slope gradient between it and the other treatments. 


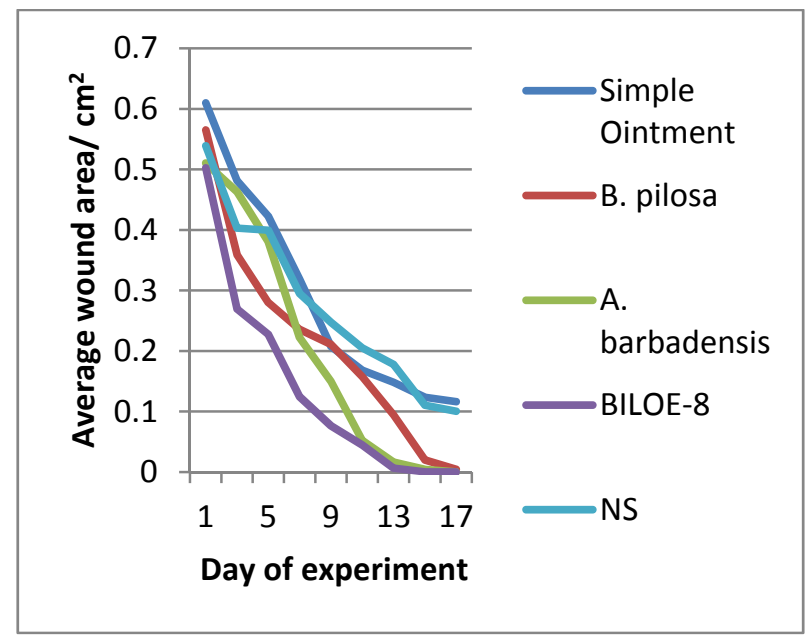

Figure 1: Relative changes in wound area for the different experimental groups over 17 days.

Activity of B. pilosa in this study was similar to that in another study (percentage wound closure at day 3 was 33.33 \pm 4.07 ) as reported by Hassan et al. [13]. This could have been due to the natural processes and inherent wound contraction activity mentioned earlier. However, percentage wound closure was lower on day 9 (74.31 \pm 3.70$)$. This could have been due to differences in concentration of the extract with respect to formulation type (solution versus ointment).

Behavior of the rate is different for the treatments. BPO appears to be more efficacious within the first 3 days of treatment as compared to $\mathrm{ABO}$ that has better efficacy from about day 5. This is not much different from results obtained from another study [20], which showed a significant difference in wound surface areas on days 15 and 20 post-injury but not at the start of the experiment, even though they used a different extract of $A$. vera (aqueous). The difference between that result and that of this study was the wound size (theirs being a $4 \mathrm{~cm}^{2}$ square and ours a circle of average area $0.5453 \mathrm{~cm}^{2}$ ). BILOE-8 shows relatively consistent reduction in wound area and rate of wound closure over time.

The graph for BILOE-8 is similar to that of BPO for the first five days, and similar to that of ABO at days 11-17. From these findings, BILOE- 8 appears to exhibit properties of both BPO and ABO. In addition, judging from the faster wound reduction rate and the shortened healing time for BILOE-8, it can be deduced that the extracts of $A$. barbadensis and B. pilosa when combined have a positive pharmacodynamic interaction as compared to when each of these extracts are used alone.

Behavior of the curve with respect to wound reduction over time for Simple Ointment and NS is similar. Also, Simple Ointment and Neomycin Sulphate have comparable results as regards rate of reduction of wound area. A study done by Hassan et al. [13]. showed comparable activity between both negative (distilled water) and positive controls (NS). Percentage wound reduction for NS at days 3 and 9 was $23.35 \pm 5.11$ and $68.66 \pm 3.57$ respectively. 
For distilled water, percentage wound reduction at days 3 and 9 was $23.35 \pm 5.11$ and $63.18 \pm 2.27$ respectively. This corresponds with the findings of this study.

This implies that neomycin sulphate cream does not play a role in wound contraction, but rather is used for its antibacterial properties which help to keep the wound free of infections that would otherwise complicate the healing process. This also indicates that the accelerated rate of reduction in wound size with the other three ointments is attributed to the extracts that they contain.

\subsection{Rate of reduction in wound area}

The rate of reduction in wound area with BILOE-8 was the fastest. Percentage wound reduction by day 17 for BILOE- 8 was $100 \%$ while the lowest was Simple Ointment $(80.89 \%$ ), which was not significantly different from NS $(81.35 \%)$ as illustrated in table1. BILOE- 8 causes greater reduction in wound area over time compared to the other treatments (Table 3).

\begin{tabular}{|l|l|l|l|l|l|}
\hline DAYS & Simple Ointment & B. pilosa & A. barbadensis & BILOE-8 & NS \\
\hline 3 & 21.03 & 36.45 & 9.23 & 46.37 & 25.23 \\
\hline 9 & 65.93 & 62.65 & 70.68 & 84.78 & 54.06 \\
\hline 17 & 80.89 & 99.19 & 99.96 & 100 & 81.35 \\
\hline
\end{tabular}

Table 3: Percentage Wound Reduction by Days 3, 9, and 17.

In addition, using one-way ANOVA, it was found that there was an extremely statistically significant difference between the wound healing effects of BILOE- 8 and those of the other treatments over the days $(p<0.0001)$ as illustrated in Table 4. This implies that BILOE-8 is the most efficacious of the five treatments used in this experiment.

\begin{tabular}{|l|l|l|}
\hline BILOE-8 vs. & Difference in effects over the days & Difference in effects with treatments \\
\hline Simple Ointment & $\mathrm{F}(8,36)=74.4167,0.1786, \mathrm{p}<0.0001$ & $\mathrm{~F}(1,36)=125.2917,0.3007, \mathrm{p}<0.0001$ \\
\hline B. pilosa & $\mathrm{F}(8,36)=60.3793,0.1751, \mathrm{p}<0.0001$ & $\mathrm{~F}(1,36)=26.3448,0.0764, \mathrm{p}<0.0001$ \\
\hline A. barbadensis & $\mathrm{F}(8,36)=74.8889,0.2022, \mathrm{p}<0.0001$ & $\mathrm{~F}(1,36)=18.4074,0.0497, \mathrm{p}=0.0001$ \\
\hline NS & $\mathrm{F}(8,36)=133.5455,0.1469, \mathrm{p}<0.0001$ & $\mathrm{~F}(1,36)=227.2727,0.2500, \mathrm{p}<0.0001$ \\
\hline
\end{tabular}

Table 4: Summary of Comparisons of BILOE-8 to the other treatments for 17 days using one-way ANOVA.

\subsection{Epithelialization and healing times for wounds of the experimental rats}

BILOE-8 exhibits the shortest epithelialization and healing times, which are not significantly different from those of A. barbadensis, as illustrated in Table 5. NS and Simple Ointment exhibit the longest times, which are also not significantly different from each other. 


\begin{tabular}{|l|l|l|}
\hline Set & Epithelialization time & Healing Time \\
\hline Simple Ointment & $9.67 \pm 1.2472(7.65,11.69)$ & $20.67 \pm 0.4714(19.91,21.43)$ \\
\hline B. pilosa & $7.33 \pm 0.4717(6.57,8.09)$ & $17.67 \pm 0.4714(16.9,18.43)$ \\
\hline A. barbadensis & $6.00 \pm 0.8165(4.68,7.32)$ & $16.67 \pm 1.1547(14.8,18.54)$ \\
\hline BILOE-8 & $5.67 \pm 0.4714(4.91,6.43)$ & $15.33 \pm 0.9428(13.81,16.86)$ \\
\hline NS & $8.00 \pm 0.8165(6.68,9.32)$ & $20.00 \pm 0.8165(18.68,21.32)$ \\
\hline
\end{tabular}

Table 5: Epithelialization and Healing Times for Wounds of 15 Experimental Rats.

In addition, epithelialization and healing times for BILOE-8 are shorter than for either A. barbadensis or B. pilosa alone. This implies that there is the possibility of a positive interaction between substances in the two extracts to produce a much greater wound reduction rate.

\subsection{Toxicity profile of Biloe-8 ointment}

Data on the toxicity of the two plants used in this experiment suggests that they are safe for topical use, with any adverse effects being extremely rare or underreported. Despite presence of anthraquinones in the extract used, no cutaneous reactions were observed in the study animals.

\section{Conclusion and Recommendations}

In conclusion, a herbal ointment (BILOE-8) from combined extracts of Aloe barbadensis and Bidens pilosa is of acceptable quality and physically stable. The ointment is effective for wound healing as it exhibits better wound healing properties compared to other treatments used in this study. Improvements should be made to BILOE-8, especially to reduce the staining properties in order to make it more cosmetically acceptable. Other topical dosage forms can be formulated and tested for comparison with ointment form. Given the proven efficacy, BILOE-8 should be considered as an alternative to neomycin for wound management.

\section{Acknowledgements}

This research work was partially funded by The Pharmaceutical Society of Uganda (PSU). We would also like to extend our gratitude to Dr John Kateregga and Mr Sadrachs of Makerere University, College of Veterinary Medicine, Animal resources, and Biosecurity for the assistance rendered during conduct of the efficacy and safety studies; and Ms Elizabeth Nalule for the statistical concepts support.

\section{Declaration of Conflict of Interest}

The Authors have no conflicting interest. 


\section{References}

1. Ghosh AK, Abdul A, McDonald S. The Role of Lymphocytes in wound healing. Br J Plast Surg 43 (1990): 655-662.

2. WHO. WHO Warns Against 'Post-Antibiotic' Era: Nature- International Weekly Journal of Science. Nature Publishing Group (2014).

3. Kumar A, Fausto M. Robbins' Basic Pathology. 8th Edition. - Philadelphia : Elsevier Inc. (2007).

4. Sudeendra BR, Shankrappa J, Shivakumal HG, et al. Formulation and Evaluation of Polyherbal Wound Treatments. Asian Journal of Pharmaceutical Sciences 2 (2007): 11-17.

5. Thomas HC. Checklist for Factors Affecting Wound Healing: Advances in Skin and Wound Care Journal. Harrisburg, Pennsylvania : Lippincott Williams and Wilkins 24 (2011): 192.

6. UCG Uganda Clinical Guidelines. Kampala : Ministry of Health (2012).

7. Unnikrishnan P. Role of Traditional Medicine in Primary Health Care: An Overview of Perspectives and Challenges. Yokohama Journal of Social Sciences 14 (2010): 57-77.

8. Government of Uganda. National Policy on Public Private Partnership in Health - Kampala (2009).

9. Wound Care Centres. Wound Care Centers. woundcarecenters.org. (2014).

10. Hinz HU, Boris, Gabbiani L. Formation and Function of Myofibroblast During Tissue Repair. Journal of Investigative Dermatology 127 (2003): 526-537.

11. Juneby Hans B. Aloe barbadensis- A Legendary Medicinal Plant (2009).

12. Rajeswari R, Umadevil M, Sharmila Rahale C, et al. Aloe vera the Miracle Plant: Its Medicinal and Traditional Uses in India. Journal of Pharmacognosy and Phytochemistry 1 (2012): 118-124.

13. Hassan Kyakulaga A, Deogratius o, Nyafuono JF, et al. Wound healing potential of the ethanolic extracts of Bidens pilosa and Ocimum suave. African Journal of Pharmacy and Pharmacology 5 (2011): 132-136.

14. Amzad HM, Al-Raqmi Khulood AS, Zawan Hamood AM, et al. Study of Total Phenol, Flavonoids Contents and Phytochemical Screening of Various Leaves Crude Extracts of Locally Grown Thymus vulgaris: Asian Pacific Journal of Tropical Biomedicine. - Nizwa, Sultanate of Oman : NCBI 3 (2013): 705-710.

15. Langley Chris, Belcher Dawn.Fast Track- Pharmaceutical Compounding and Dispensing - London: Pharmaceutical Press (2008).

16. BP. British Pharmacopoeia. London : Crown Publishers (2007).

17. Aulton ME. Pharmaceutics- The Science of Dosage Form and Design 2nd Edition - London : ChurchillLivingstone (2002).

18. Drugs.com http://www.drugs.com (2014).

19. BP. British Pharmacopoeia. London : Crown Publishers (1988).

20. Oryan A, Naeini AT, Nikahval B, et al. Effect of Aqueous Extract of Aloe vera on Experimental Cutaneous Wound Healing in a Rat: Veterinarski Arhiv Journal 80 (2010): 509-522. 
Citation: Sandra Namunana, Stephen Lutoti, Grace Nyamaizi, Gerald Agaba, Irene Apun, Charles Ssebunnya, Gladys Mercy Tenywa, Raphael Wangalwa, Bruhan Kaggwa, Pakoyo Fadhiru Kamba, David Musoke-Muweke, Patrick Ogwang Engeu. Formulation, Development and Validation of a Wound Healing Herbal Ointment from Extracts of Bidens pilosa and Aloe barbadensis. J Pharm Pharmacol Res 2 (2018): 032-038.

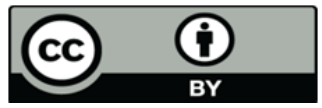

